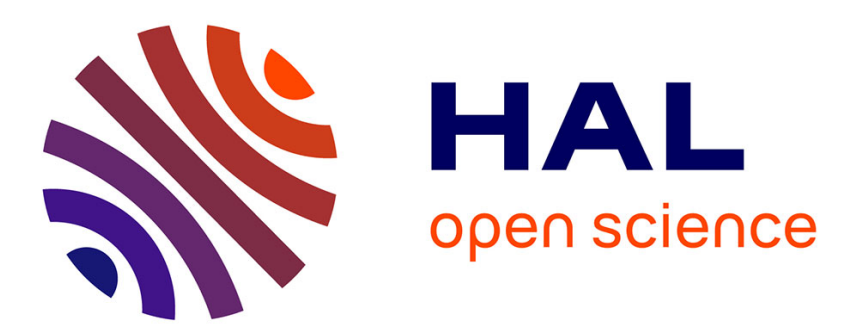

\title{
Polynomial modal method for analysis of the coupling between a gap plasmon waveguide and a square ring resonator
}

Kofi Sényo Edee

\section{- To cite this version:}

Kofi Sényo Edee. Polynomial modal method for analysis of the coupling between a gap plasmon waveguide and a square ring resonator. Journal of Applied Physics, 2017, 122 (15), pp.153102 153101. 10.1063/1.4999025. hal-01658978

\section{HAL Id: hal-01658978 \\ https://hal.uca.fr/hal-01658978}

Submitted on 8 Dec 2017

HAL is a multi-disciplinary open access archive for the deposit and dissemination of scientific research documents, whether they are published or not. The documents may come from teaching and research institutions in France or abroad, or from public or private research centers.
L'archive ouverte pluridisciplinaire HAL, est destinée au dépôt et à la diffusion de documents scientifiques de niveau recherche, publiés ou non, émanant des établissements d'enseignement et de recherche français ou étrangers, des laboratoires publics ou privés. 
Polynomial modal method for analysis of the coupling between a gap plasmon waveguide and a square ring resonator

Kofi Edee

Citation: Journal of Applied Physics 122, 153102 (2017); doi: 10.1063/1.4999025

View online: http://dx.doi.org/10.1063/1.4999025

View Table of Contents: http://aip.scitation.org/toc/jap/122/15

Published by the American Institute of Physics

Articles you may be interested in

Voltage tunable plasmon propagation in dual gated bilayer graphene

Journal of Applied Physics 122, 153101 (2017); 10.1063/1.5007713

\section{Scilight}

Sharp, quick summaries illuminating the latest physics research 


\title{
Polynomial modal method for analysis of the coupling between a gap plasmon waveguide and a square ring resonator
}

\author{
Kofi Edee ${ }^{a)}$ \\ Université Clermont Auvergne, Institut Pascal, BP 10448, F-63000 Clermont-Ferrand, France and CNRS, \\ UMR 6602, Institut Pascal, F-63177 Aubière, France
}

(Received 4 August 2017; accepted 2 October 2017; published online 17 October 2017)

\begin{abstract}
When a Metal-Insulator-Metal waveguide is coupled with a ring resonator, one expects, for a given integer referring to cavity mode number, exactly one resonance phenomenon. However, numerical simulations of the spectrum of the transmission of the structure highlight two resonances around certain values of the resonance wavelength. This phenomenon was numerically studied thanks to a Finite Difference Time Domain method. Until now, and to my knowledge, this phenomenon is linked to a correlation between the standing waves forming in the ring at the resonance wavelength and the faces and corners of the ring. In this document, I present a new physical analysis and interpretation of the transmission spectrum anomaly of the square ring resonator. By using an efficient polynomial modal method, I show that this phenomenon is due to the interference of hybrid modes of the superstructure. Published by AIP Publishing. https://doi.org/10.1063/1.4999025
\end{abstract}

\section{INTRODUCTION}

Classical circular waveguides' ring resonators ${ }^{1}$ have been widely studied. They have been used in photonics integrated circuits and dense wavelength division multiplexing optical communication systems such as filters, add-drop couplers, and a wavelength-division multiplexer. In 2007, a square ring resonator (SRR) was studied by Hosseini and Massoud. ${ }^{2}$ This SRR can be used as an ultracompact directional add-drop coupler between two parallel waveguides. Several years later, other authors extended the scope of this system to realize, for example, plasmon flow control in $\mathrm{T}$ splitters and waveguide cross junctions. ${ }^{3}$ Recently in 2015, a novel surface plasmon based square-shaped ring resonator with a bending Metal-Insulator-Metal (MIM) waveguide at the optical spectral range was investigated by Yan et al. in Ref. 4. The feature of this structure was often analyzed thanks to a finite difference in the time domain (FDTD) method and its transmission spectrum may highlight an anomaly. This anomaly was observed and explained first in Ref. 2 by suggesting a correlation between the standing waves forming in the ring at the resonance $\lambda_{g}$ and the faces and corners of the ring. Although these observations and analysis seem relevant, it is possible to deepen and complete them by a modal analysis of the structure. Thus I present in this paper another interpretation of this phenomenon which relies on the multimode character of this type of structure. My analysis is based on the propagation of plasmons through the interference of two modes. These two modes result from the coupling between the waveguide bus and the resonator. Since the feature of devices at hand is linked to a plasmon resonance phenomenon, it may be successfully treated as an eigenvalue problem with specific boundary conditions, i.e., a boundary value problem. This boundary value problem is efficiently solved throughout a polynomial modal method

a)kofi.edee@uca.fr based on Gegenbauer expansion (MMGE) ${ }^{5-8}$ I first show that this numerical scheme allows avoidance of any uncertainties in the numerical model. A new physical analysis and interpretation of the transmission spectrum anomaly is then suggested.

\section{STATEMENT OF THE PROBLEM}

Figure 1 shows a typical filter based on the plasmonic filter structure. It consists of a MIM waveguide and a SRR. At the resonance wavelength $\lambda_{g}$, one expects that the fundamental mode of the MIM waveguide, whose propagation constant is denoted by $\gamma_{0}$, may be coupled to the ring and thus the flow of energy may be exchanged between resonator modes. By neglecting edge effects and evanescent modes contributions, the resonance condition is then written as

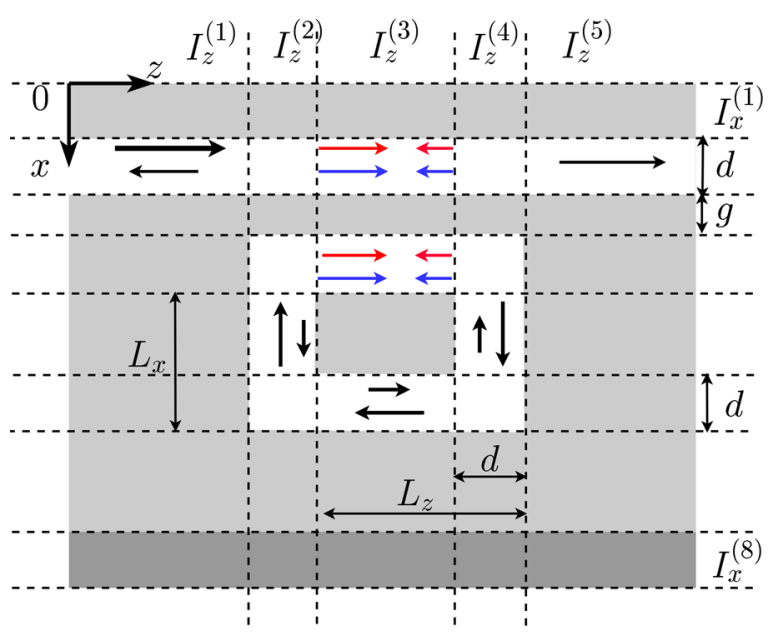

FIG. 1. Schematic of a plasmonic rectangular MIM resonator. Illustration of the staircase concatenation of the MIM resonator according to the $(\mathrm{Oz})$ and $(O x)$ directions. The structure is divided into five layers $I_{z}^{(k)}, k=1: 5$ in the $(O z)$ direction and height intervals $I_{x}^{(k)}, k=1: 8$ in the $(O x)$ direction. The layers $I_{z}^{(1)}$ (resp. $I_{z}^{(5)}$ ) correspond to the input (resp. output) waveguide bus. 


$$
2\left(L_{x}+L_{z}\right)=n_{g} \lambda_{g}
$$

$\lambda_{g}=\lambda / n_{\text {eff }}$ is the guide wavelength. $n_{\text {eff }}=\gamma_{0} / k_{0}$ denotes the effective index and $\lambda$ is the free space wavelength. In this equation (1) the integer $n_{g}$ stands for cavity mode number. $k_{0}=2 \pi / \lambda$ is the wave number. Referring to Eq. (1), for a given value of the integer $n_{g}$ only one resonance is expected. However, numerical simulations of the spectrum of the transmission of the structure reported in Fig. 2 highlight two resonances around certain values of $\lambda_{g}$. I present here an interpretation of this phenomenon which relies on the multimode character of this type of structure. My analysis is based on the propagation of plasmons through the interference of two modes, resulting from the coupling between the waveguide bus and the resonator.

\section{POLYNOMIAL MODAL METHOD OF MIM WAVEGUIDE MODELLING}

First of all we must ensure that there are no uncertainties in our polynomial numerical model. For that purpose, we are interested in the effective index $\gamma_{0} / k_{0}$ of a metal-insulatormetal waveguide, which gives us a good test of robustness of our numerical scheme. $k_{0}$ denotes the wavenumber. The dielectric is assumed to be air with relative permittivity $\varepsilon=1$. The metal is a silver whose complex relative permittivity $\varepsilon_{\text {silver }}$ is characterized through the Drude model

$$
\varepsilon_{\text {silver }}(\omega)=\varepsilon_{\infty}-\frac{\omega_{p}}{\omega(\omega+i \Gamma)},
$$

where the bulk plasma frequency $\omega_{p}=1.38 \times 10^{16}$, the dumping frequency of the oscillations $\Gamma=2.73 \times 10^{13}$, and $\varepsilon_{\infty}=3.7=\varepsilon_{\text {silver }}(\omega=\infty) . \omega$ is the angular frequency. For our numerical illustration, the gap $d$ of the waveguide is set to $50 \mathrm{~nm}$. The propagation constant $\gamma_{0}$ of the fundamental mode obeys the following dispersion relation:

$$
\tanh \left(\frac{d}{2} \sqrt{\gamma_{0}^{2}-k_{0}^{2} \varepsilon_{\text {air }}}\right)=-\frac{\varepsilon_{\text {air }}}{\varepsilon_{\text {silver }}} \sqrt{\frac{\gamma_{0}^{2}-k_{0}^{2} \varepsilon_{\text {silver }}}{\gamma_{0}^{2}-k_{0}^{2} \varepsilon_{\text {air }}}} .
$$

Using this relation Eq. (3), the effective index $\gamma_{0} / k_{0}$ of the fundamental $T M_{0}$ for $\lambda=1161 \mathrm{~nm}$ is equal to

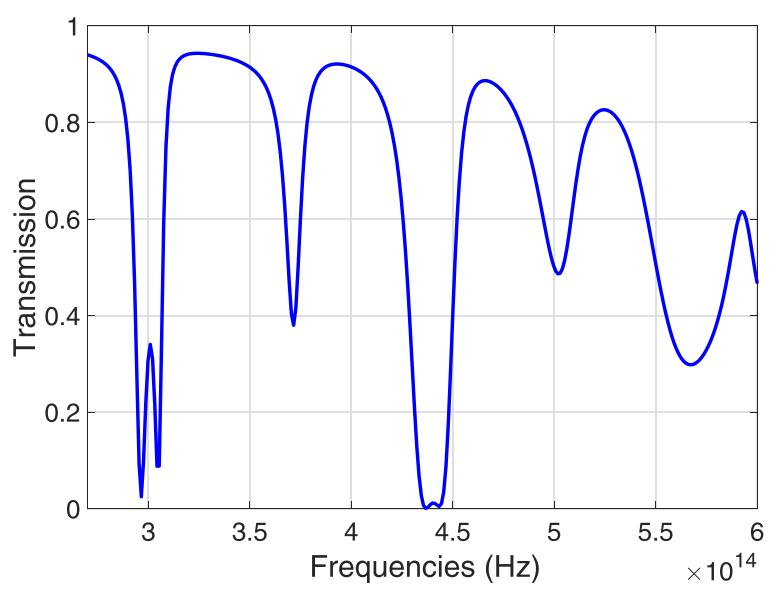

FIG. 2. Transmission spectrum of the MIM resonator. Numerical parameters: $L_{x}=L_{z}=725 \mathrm{~nm}, g=25 \mathrm{~nm}, d=50 \mathrm{~nm}$.

$$
\gamma_{0} / k_{0}=1.381850741177741-0.003033434481031 i .
$$

For the numerical evaluation through the subsectional polynomial basis approach MMGE, ${ }^{5-8}$ the slot waveguide is subdivided into four homogeneous domains (intervals) $\left(I_{x}^{(1)}, I_{x}^{(2)}\right.$, $\left.I_{x}^{(3)}, I_{x}^{(4)}\right)$ along the $(O x)$ axis with width $\left(e_{s u b}, d, e_{\text {sup }}, e_{p m l}\right)$ $=(300,50,300,50) \mathrm{nm}$ and relative permittivity $\left(\varepsilon_{\text {silver }}(\omega)\right.$, $\left.\varepsilon_{\text {air }}, \varepsilon_{\text {silver }}(\omega) \varepsilon_{\text {silver }}(\omega)\right)$. See Fig. 3. The eigenvalue equation (5)

$$
\left[\varepsilon(x) \partial_{1} \frac{1}{\varepsilon(x)} \partial_{1}+k_{0}^{2} \varepsilon(x)\right] H_{y}(x)=\gamma^{2} H_{y}(x),
$$

is then solved in the TM polarization case and the eigenvalue corresponding to the effective index of the fundamental mode $\gamma_{0} / k_{0}$ is computed. We present in Table I the convergence of the result in the case of Gegenbauer polynomials $G_{n}^{\Lambda}(x)$ with $\Lambda=0.5$ with respect to a parameter Dim. The parameter Dim denotes the number of polynomial basis functions allowing to describe the electromagnetic field on $I_{x}=\cup_{k=1: 4} I_{x}^{(k)}$.The interval $I_{x}^{(4)}$ corresponds to the Perfectly Matched Layer (PML), and in all the following computations, we set the PML thickness to $e_{p m l}=50 \mathrm{~nm}$, and its parameters $\chi+i \eta=10-10 i$. $\mathrm{Cf}^{7}$ for the implementation of the PML in the present polynomial modal method throughout a complex coordinates transform. An accuracy of $10^{-7}$ is achieved for $\operatorname{Dim}=12$.

\section{MODAL ANALYSIS OF THE SRR: INTERFERENCE OF HYBRID MODES}

In order to compute the transmission of the structure, throughout the polynomial modal method based on Gegenbauer polynomials $G_{n}^{\Lambda}(x)$ expansion, the structure is divided into nine intervals $I_{x}^{(k)}, k=1: 8$ in the $(O x)$ direction and five layers $I_{z}^{(k)}, k=1: 5$ in the $(O, z)$ direction (Fig. 3). In each layer $I_{z}^{(k)}$, the general solution $\phi^{(k)}(x, z)$ representing the component

$$
H_{y}^{(k)}(x, z)=H_{y}^{(k)+}(x, z)+H_{y}^{(k)-}(x, z),
$$

is expressed as the linear combination of:

- forward waves propagating along increasing values of $z$

$$
\begin{aligned}
H_{y}^{(k)+}(x, z) & =\sum_{q} A_{q}^{(k)} \exp \left(-i k_{0} \gamma_{q}^{(k)} z\right) \sum_{n} \psi_{n q}^{(k)} G_{n}^{\Lambda}(x) \\
& =\sum_{q} H_{q}^{(k)+}(x, z) .
\end{aligned}
$$

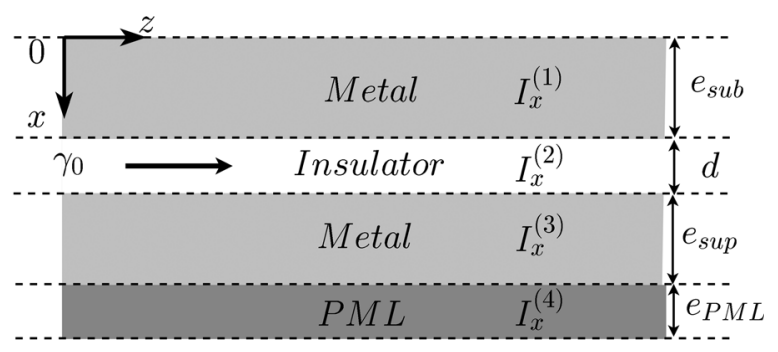

FIG. 3. Illustration of the numerical configuration for the modal analysis of MIM waveguide. The structure is subdivided into four homogeneous domains (intervals) $\left(I_{x}^{(1)}, I_{x}^{(2)}, I_{x}^{(3)}, I_{x}^{(4)}\right)$ along the $(O x)$ axis with the thickness $\left(e_{s u b}, d, e_{\text {sup }}, e_{p m l}\right)$. 
TABLE I. Convergence of the effective index $\gamma_{0} / k_{0}$ of the mode $T M_{0}$ computed thanks to modified Gegenbauer polynomials $G_{n}^{\Lambda}(x)$ with $\Lambda=0.5$. Numerical parameters: $d=50 \mathrm{~nm}, e_{\text {sub }}=e_{\text {sup }}=300 \mathrm{~nm}, e_{p m l}=50 \mathrm{~nm}, \chi$ $-i \eta=10-10 i, \lambda=1161 \mathrm{~nm}$.

\begin{tabular}{lc}
\hline \hline Dim & effective index \\
\hline 6 & $1.381866066132478-0.003034206565412 \mathrm{i}$ \\
12 & $1.381850837595450-0.003033438956998 \mathrm{i}$ \\
18 & $1.381850741448333-0.003033436438067 \mathrm{i}$ \\
24 & $1.38185074138777-0.003033436451930 \mathrm{i}$ \\
30 & $1.381850741414873-0.003033436469400 \mathrm{i}$ \\
36 & $1.381850741426127-0.003033436500549 \mathrm{i}$ \\
42 & $1.381850741621650-0.003033436337988 \mathrm{i}$ \\
48 & $1.381850741453263-0.003033436384211 \mathrm{i}$ \\
54 & $1.381850741586496-0.003033436562868 \mathrm{i}$ \\
60 & $1.381850741240158-0.003033436406518 \mathrm{i}$ \\
\hline \hline
\end{tabular}

- backward waves propagating along decreasing values of $z$

$$
\begin{aligned}
H_{y}^{(k)-}(x, z) & =\sum_{q} B_{q}^{(k)} \exp \left(i k_{0} \gamma_{p}^{(k)} z\right) \sum_{n} \psi_{n q}^{(k)} G_{n} \Lambda(x) \\
& =\sum_{q} H_{q}^{(k)-}(x, z) .
\end{aligned}
$$

Layers $I_{z}^{(2)}, I_{z}^{(3)}$, and $I_{z}^{(4)}$ correspond to the coupling region. In the case when standing-waves are excited and live in the cavity, the eigenvalues $\gamma_{p}^{(3)}$ may provide a good estimation of their effective indices in the $(O, z)$ direction. The major part of the coupling region may be considered as a superstructure that consists of three parallel slot waveguides $I_{x}^{(2)}, I_{x}^{(4)}$, and $I_{x}^{(6)}$ with width $d=50 \mathrm{~nm}$. The coupled modes theory suggests that when the distance between these three waveguides is large enough, there is no interaction between them and the eigenvalues of the whole superstructure are just those of each structure: the eigenvalues are three-degree degenerate; otherwise, the coupling removes or lifts the degeneracy involving a modification of the modes of each waveguide eigenmode called hybrid modes. See Fig. 4. For this example, the distance between $I_{x}^{(4)}$ and $I_{x}^{(6)}$ is set to $L_{z}-d$ $=675 \mathrm{~nm}$. Since the distance between $I_{x}^{(4)}$ and $I_{x}^{(6)}$ is large enough, one of the initial modes of the isolated waveguide persists $\gamma_{2}^{(3)}=1.3868-0.0028 i$ in the waveguide $I_{x}^{(6)}$ for all values of $g$. However, two hybrid modes $\gamma_{1}^{(3)}$ and $\gamma_{3}^{(3)}$ appear in the coupled waveguides $I_{x}^{(2)}$ and $I_{x}^{(4)}$ for small values of $g$. For example, for $g=20 \mathrm{~nm}, \gamma_{1}^{(3)}=1.2867-0.0016 i$ and $\gamma_{3}^{(3)}=1.6035-0.0065 i$. As a starting point of our analysis, consider in the input port of the coupling layer $I_{z}^{(3)}$, the normalised forward power $\tau_{q}=P_{q} / P_{i n c}(q=1,3)$ associated with the hybrid modes $\gamma_{1}^{(3)}$ and $\gamma_{3}^{(3)}$ where

$$
P_{\text {inc }}=\operatorname{Real}\left[\sum_{q} \int_{x} E_{q}^{(3)+}(x, 0) \operatorname{conj}\left(H_{q}^{(3)+}(x, 0)\right) d x\right]
$$

and

$$
P_{q}=\operatorname{Real}\left[\int_{x} E_{q}^{(3)+}(x, 0) \operatorname{conj}\left(H_{q}^{(3)+}(x, 0)\right) d x\right] .
$$

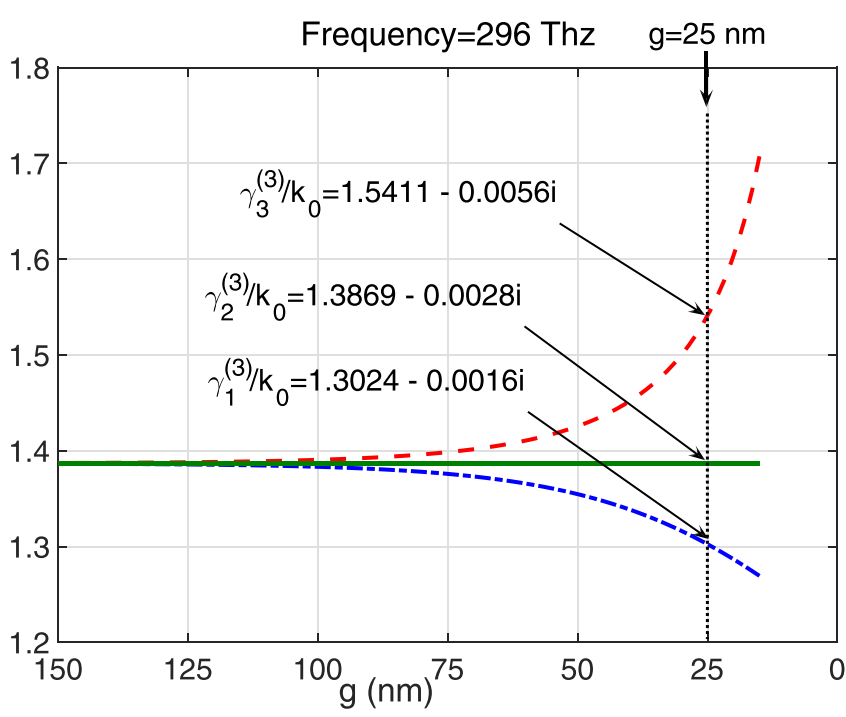

FIG. 4. Illustration of the lift of degeneracy of eigenmodes of the superstructure for $g \in[15,145] \mathrm{nm}$. Numerical parameters: frequency $=296 \mathrm{THz}$, $L_{x}=725 \mathrm{~nm}, d=50 \mathrm{~nm}$.

Figure 5 presents the behaviour of $\tau_{1,3}$ with respect to different values of the frequency (freq $=\omega / 2 \pi \in[2.7$, 3.3] THz). The transmission $|t|^{2}$ is also reported in this figure. One can remark that the energy exchange takes place between both hybrid modes. The propagated energy is injected inside the ring cavity after passing through the coupling region where the energy is exchanged between weakly coupled hybrid modes. For certain frequencies, 296.6 THz, $301.2 \mathrm{THz}$, and $304.4 \mathrm{THz}$, these modes carry power of equal intensity and interfere destructively in the waveguide bus inducing extremely weak field in the transmission, or constructively, inducing excitation of the output port. Figures 6, 7, and 8 present the modulus of the magnetic field $\mathrm{Hy}(\mathrm{x}, \mathrm{z})$ at the resonance frequencies $296.6 \mathrm{THz}, 301.2 \mathrm{THz}$, and $304.4 \mathrm{THz}$. At all these frequencies, the ring resonator is always excited, but the interference process does not cancel the field in the waveguide bus at $301.2 \mathrm{THz}$ contrary to the frequencies $296.6 \mathrm{THz}$ and $304.4 \mathrm{THz}$ where only a weak field is induced in the transmission port.

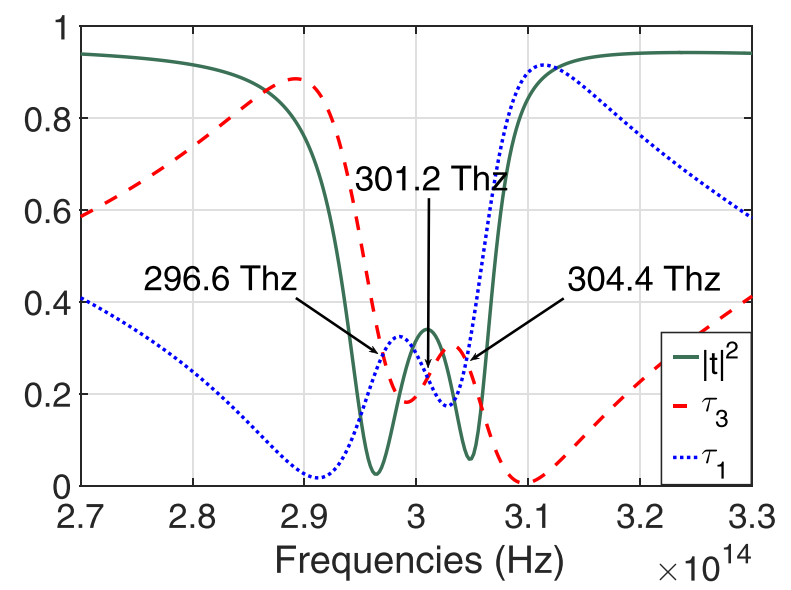

FIG. 5. Normalized forward power spectra $\tau_{1}$ (resp. $\tau_{3}$ ) associated with the eigenmode $\gamma_{1}^{(3)}\left(\right.$ resp. $\left.\gamma_{3}^{(3)}\right)$ for $g=25 \mathrm{~nm}$. Numerical parameters: $L_{x}=L_{z}$ $=725 \mathrm{~nm}, d=50 \mathrm{~nm}$ 


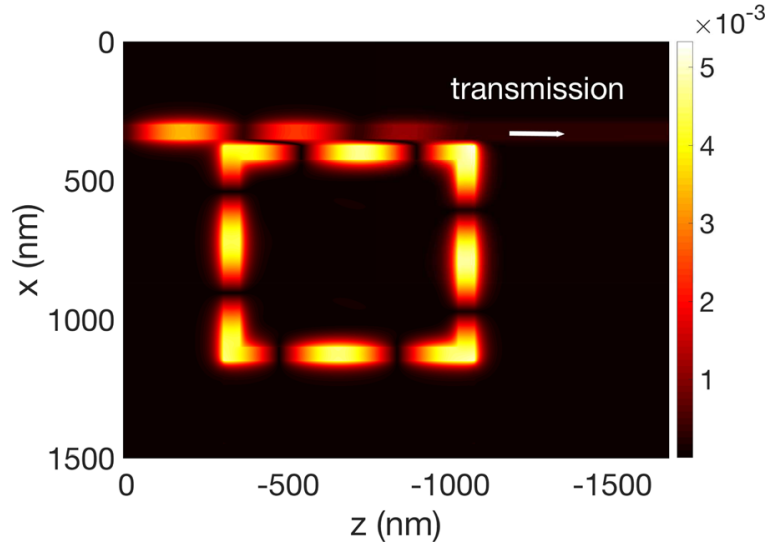

FIG. 6. Modulus of $H_{y}(x, z)$ at frequency $296.6 \mathrm{THz}$, for $g=25 \mathrm{~nm}$. Numerical parameters: $L_{x}=L_{z}=725 \mathrm{~nm}, g=25 \mathrm{~nm}, d=50 \mathrm{~nm}$.

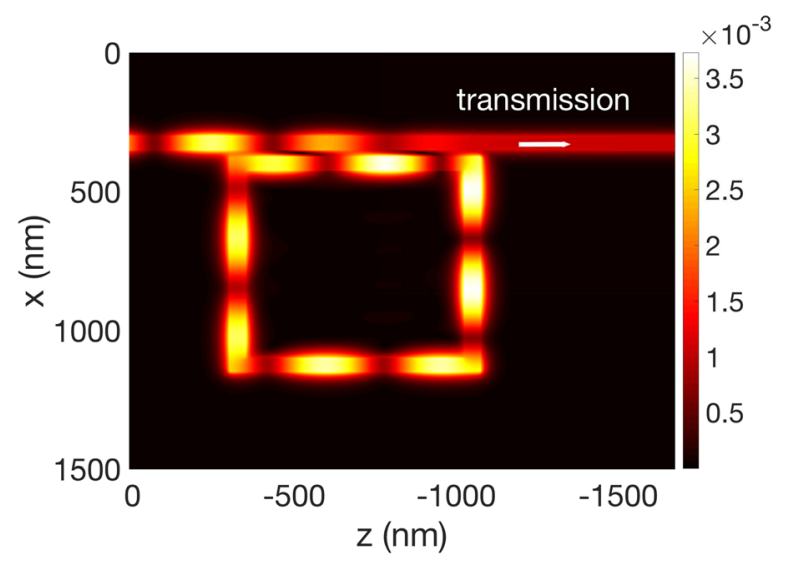

FIG. 7. Modulus of $H_{y}(x, z)$ at frequency $301.2 \mathrm{THz}$, for $g=25 \mathrm{~nm}$. Numerical parameters: $L_{x}=L_{z}=725 \mathrm{~nm}, g=25 \mathrm{~nm}, d=50 \mathrm{~nm}$.

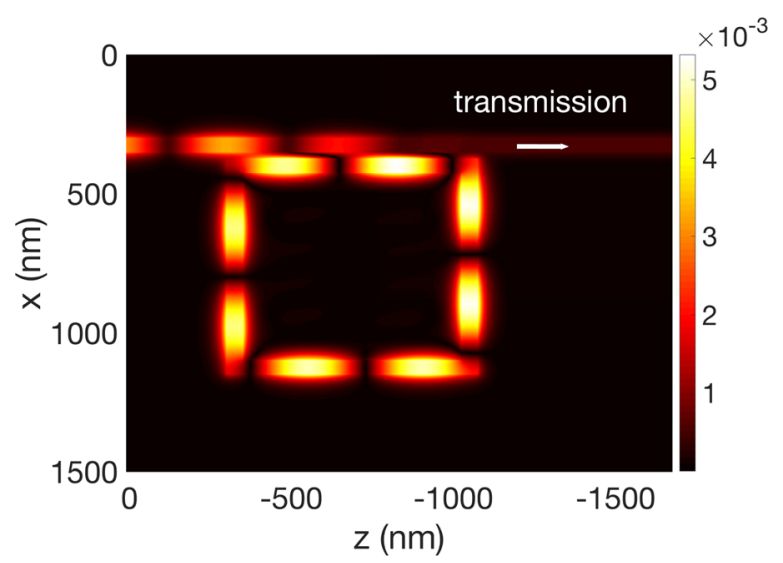

FIG. 8. Modulus of $H_{y}(x, z)$ at frequency $304.4 \mathrm{THz}$, for $g=25 \mathrm{~nm}$. Numerical parameters: $L_{x}=L_{z}=725 \mathrm{~nm}, g=25 \mathrm{~nm}, d=50 \mathrm{~nm}$.

\section{ELECTROMAGNETICALLY INDUCED TRANSPARENCY BEHAVIOUR OF THE SRR}

Let us first consider a set of four classical oscillators coupled through a weak spring with eigen-frequencies $\omega_{1} / 2 \pi, \omega_{2} / 2 \pi, \omega_{3} / 2 \pi, \omega_{4} / 2 \pi$ and damping constants $\gamma_{1}, \gamma_{2}$, $\gamma_{3}$, and $\gamma_{4}$. The coupling coefficients of these four oscillators are denoted by $\nu_{i j}, i, j \in\{1,2,3,4\}$. We assume that they are submitted to two driving forces with the same circular frequency $\omega$ and amplitudes $a_{1}$ and $a_{4}$. If we denote by $x_{i}(i=1,2,3,4)$ the amplitudes of these oscillators, then it can be easily demonstrated that the system of differential equations describing the motion is given by

$$
\left\{\begin{array}{l}
\frac{d^{2} x_{1}}{d t^{2}}+\gamma_{1} \frac{d x_{1}}{d t}+\omega_{1} x_{1}+\nu_{12} x_{2}+\nu_{13} x_{3}+\nu_{14} x_{4}=a_{1} e^{i \omega t} \\
\frac{d^{2} x_{2}}{d t^{2}}+\gamma_{2} \frac{d x_{2}}{d t}+\omega_{2} x_{2}+\nu_{21} x_{1}+\nu_{23} x_{3}+\nu_{24} x_{4}=0 \\
\frac{d^{2} x_{3}}{d t^{2}}+\gamma_{3} \frac{d x_{3}}{d t}+\omega_{3} x_{3}+\nu_{31} x_{1}+\nu_{32} x_{2}+\nu_{34} x_{4}=0 \\
\frac{d^{2} x_{4}}{d t^{2}}+\gamma_{4} \frac{d x_{4}}{d t}+\omega_{4} x_{4}+\nu_{41} x_{1}+\nu_{42} x_{2}+\nu_{43} x_{3}=a_{4} e^{i \omega t}
\end{array}\right.
$$

The steady-state harmonic solutions for the displacements $x_{i}(t)$ are

$$
x_{i}(t)=c_{i} e^{i \omega t} .
$$

It is then possible to exhibit a specific shape that combines Lorentz and Fano resonances and an interference phenomenon of some of these oscillators for suitable chosen values of the parameters of this coupled system.

Figures 9, 10, and 11 present the spectral responses $\left|c_{2}(\omega)\right|$ and $\left|c_{3}(\omega)\right|$ for different values of the parameters of $\gamma_{2}$ and $\gamma_{3}$. The remaining numerical parameters are: $\omega_{1} / 2 \pi$ $=2.93 \mathrm{~s}^{-1}, \omega_{2} / 2 \pi=2.96 \mathrm{~s}^{-1}, \omega_{3} / 2 \pi=3.04 \mathrm{~s}^{-1}, \omega_{4} / 2 \pi$ $=3.09 \mathrm{~s}^{-1}, \gamma_{1}=\gamma_{4}=0.2, \nu_{12}=\nu_{21}=\nu_{23}=\nu_{32}=\nu_{34}=\nu_{43}$ $=0, \nu_{13}=\nu_{31}=\nu_{24}=\nu_{42}=0.05, a_{1}=a_{4}=1, a_{2}=a_{3}=0$. In Fig. 9, $\gamma_{2}=\gamma_{3}=0.5$, while in Fig. 10, $\gamma_{2}=\gamma_{3}=0.4$ and these parameters are equal to $\gamma_{2}=\gamma_{3}=0.3$ in Fig. 11. We remark that the shape of the curves around the interference area is essentially driven by the values of the damping constants $\gamma_{2}$ and $\gamma_{3}$ of oscillators 2 and 3. Therefore, the number

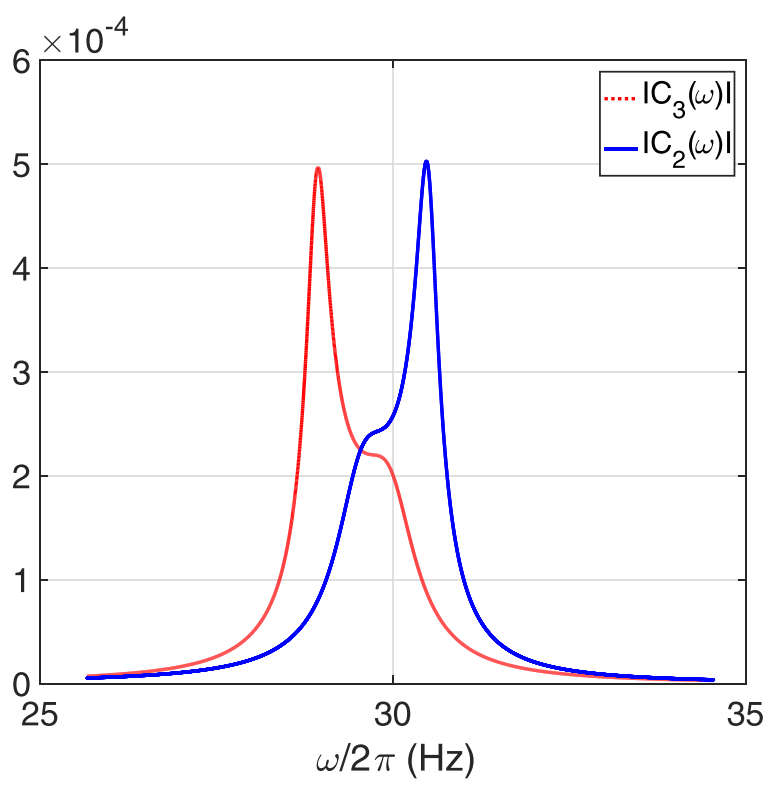

FIG. 9. Fano resonance illustration with two coupled oscillators. Numerical parameters: $\omega_{1} / 2 \pi=2.93, \omega_{2} / 2 \pi=2.99, \omega_{3} / 2 \pi=3.04, \omega_{4} / 2 \pi=3.09, \gamma_{1}$ $=\gamma_{4}=0.2, \gamma_{2}=\gamma_{3}=0.5, \nu_{13}=\nu_{31}=\nu_{14}=\nu_{41}=0.05, a_{1}=1=a_{4}=1$. 


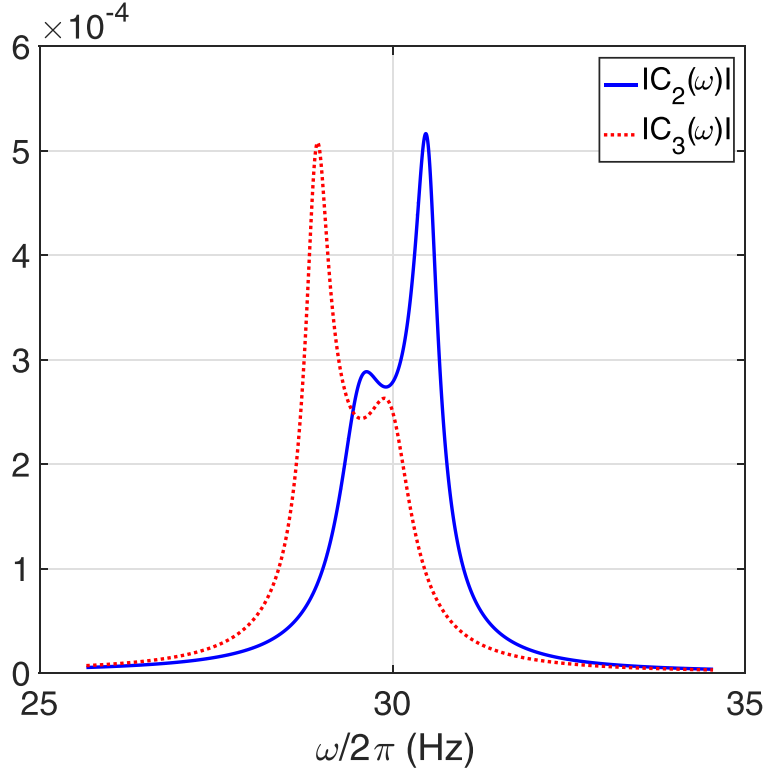

FIG. 10. Fano resonance illustration with two coupled oscillators. Numerical parameters: $\omega_{1} / 2 \pi=2.93, \omega_{2} / 2 \pi=2.99, \omega_{3} / 2 \pi=3.04, \omega_{4} / 2 \pi=3.09, \gamma_{1}$ $=\gamma_{4}=0.2, \gamma_{2}=\gamma_{3}=0.4, \nu_{13}=\nu_{31}=\nu_{14}=\nu_{41}=0.05, a_{1}=1=a_{4}=1$.

of the interference area strongly depends on these damping constants.

Let us secondly consider the problem of the coupling between the MIM waveguide and the ring resonator. It is possible to highlight an analogy between the behavior of the previous four classical coupled oscillators and the anomaly observed in the case of the SRR. To do so, let us define in Eq. (13), at the bottom interface of layer 3, a local coefficient of reflection $r_{1}^{(3)}$ (resp. $r_{3}^{(3)}$ ) of the eigenmodes corresponding to the eigenvalues $\gamma_{1}^{(3)}$ (resp. $\gamma_{3}^{(3)}$ )

$$
r_{1}^{(3)}(\omega)=\frac{A_{1}^{(3)}(\omega)}{B_{1}^{(3)}(\omega)}, \quad \text { and } \quad r_{3}^{(3)}(\omega)=\frac{A_{3}^{(3)}(\omega)}{B_{3}^{(3)}(\omega)}
$$

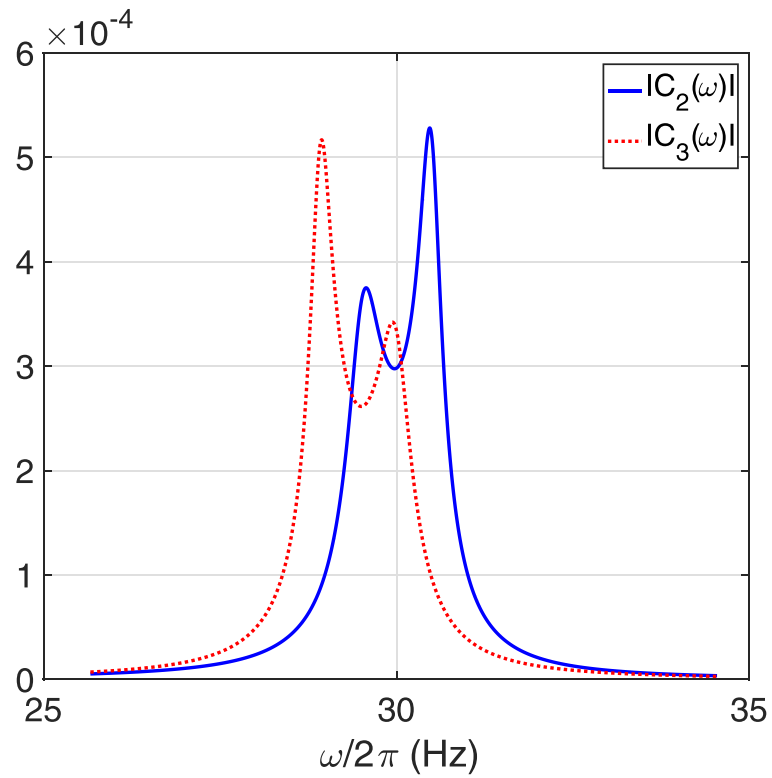

FIG. $11 . \omega_{1} / 2 \pi=2.93, \omega_{2} / 2 \pi=2.99, \omega_{3} / 2 \pi=3.04, \omega_{4} / 2 \pi=3.09, \gamma_{1}$ $=\gamma_{4}=0.2, \gamma_{2}=\gamma_{3}=0.3, \nu_{13}=\nu_{31}=\nu_{14}=\nu_{41}=0.05, a_{1}=1=a_{4}=1$.

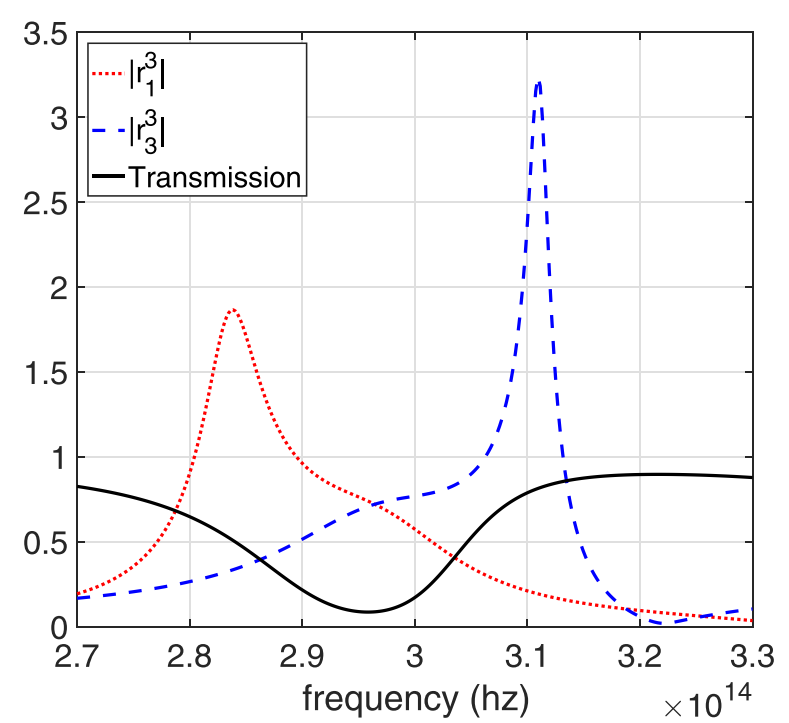

FIG. 12. Transmission spectra $(\omega / 2 \pi \in[260,340] \mathrm{THz})$ of the MIM resonator for $g=15$. Other Numerical parameters: $L_{x}=L_{z}=725 \mathrm{~nm}, d=50 \mathrm{~nm}$.

Figures 12,13 , and 14 present the behavior of $\left|r_{1,3}^{(3)}\right|$ with respect to different values of the frequency $(f=\omega / 2 \pi$ $\in[2.6,3.5] \mathrm{THz})$ and for different values of the parameters $g: g=15$ in Fig. 12, $g=20$ in Fig. 13, and $g=25$ in Fig. 14, The transmission $|t|^{2}$ is also reported in these figures. The spectral responses of both eigenmodes are similar to that previously presented in Figs. 9, 10, and 11. By tuning the parameter $g$ which leads to the suppression of extinction at the resonance frequency around the ring mode resonance frequency, one may exhibit a specific shape that combines Lorentz and Fano resonances and an interference phenomenon of some eigenmodes of the coupled system. More generally, Fig. 15 presents the behaviour of the transmission spectrum for different values of $g$. One can remark that transparency may be electromagnetically induced in the transmission. The Electromagnetically Induced

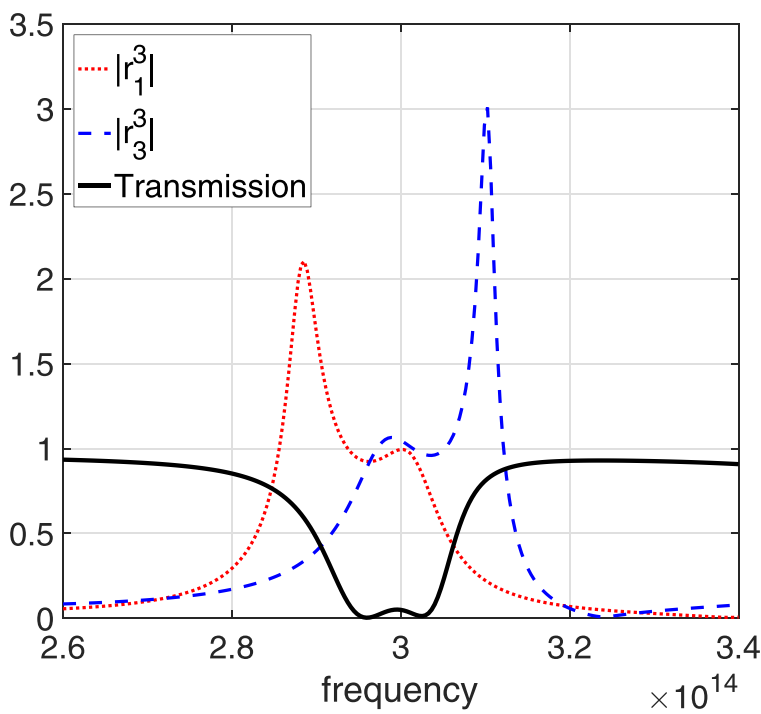

FIG. 13. Transmission spectra $(\omega / 2 \pi \in[260,340] \mathrm{THz})$ of the MIM resonator for $g=20$. Other Numerical parameters: $L_{x}=L_{z}=725 \mathrm{~nm}, d=50 \mathrm{~nm}$. 


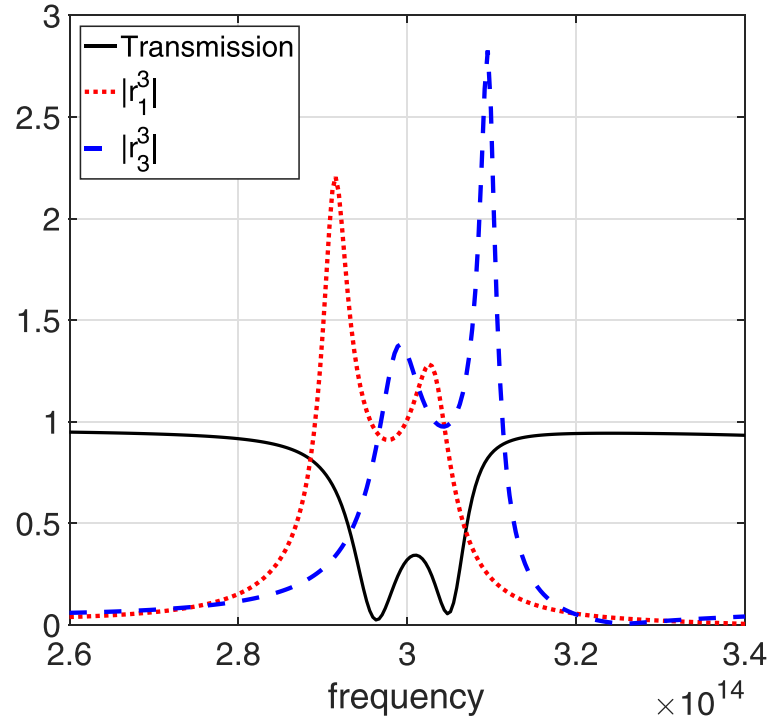

FIG. 14. Transmission spectra $(\omega / 2 \pi \in[260,340] \mathrm{THz})$ of the MIM resonator for $g=25$. Other Numerical parameters: $L_{x}=L_{z}=725 \mathrm{~nm}, d=50 \mathrm{~nm}$.

Transparency (EIT) phenomenon ${ }^{9,10}$ is well known to be linked to an interference phenomenon. This phenomenon cannot be explained through the resonance condition of Eq. (1).

\section{CONCLUSION}

In the present work, the anomaly highlighted in the transmission spectrum of a square ring resonator is well explained thanks to a modal analysis. We demonstrate that these anomalies are linked to the interferences of hybrid modes of the superstructure. These modes have been efficiently expanded on a series sum of Gegenbauer polynomials and computed as eigenfunctions of a boundary value problem. This polynomial modal method is very stable, accurate, and converges very rapidly. Our study clearly shows that the split of the resonance in the transmission spectrum is linked

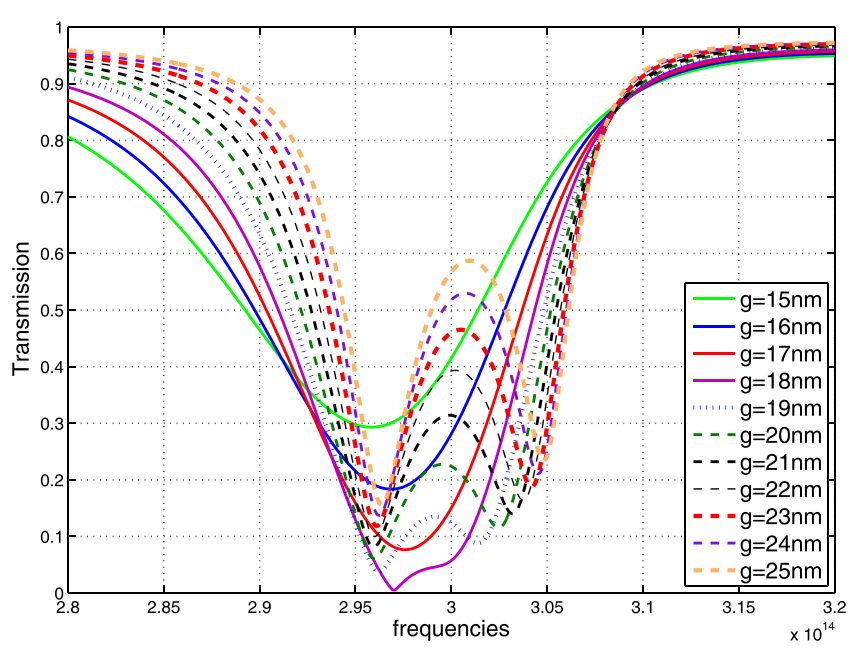

FIG. 15. Transmission spectra $(\omega / 2 \pi \in[260,340] \mathrm{THz})$ of the MIM resonator for different values of $g$. Other Numerical parameters: $L_{x}=L_{z}$ $=725 \mathrm{~nm}, d=50 \mathrm{~nm}$.

to an EIT phenomenon and this phenomenon cannot be well explained with the resonance condition of the cavity of the square ring resonator.

${ }^{1}$ S. Xiao, L. Liu, and M. Qiu, Opt. Express 14, 2932-2937 (2006).

${ }^{2}$ A. Hosseini and Y. Massoud, Appl. Phys. Lett. 90, 181102 (2007).

${ }^{3}$ J. Liu, G. Fang, H. Zhao, Y. Zhang, and S. Liu, J. Phys. D: Appl. Phys. 43, 055103 (2010).

${ }^{4}$ Y.-L. Yan, G. Fu, Y. Zhang, S.-X. Gong, and X. Chen, Prog. Electromagn. Res. Lett. 51, 39 (2015).

${ }^{5}$ K. Edee, J. Opt. Soc. Am. A 28, 2006-2013 (2011).

${ }^{6}$ K. Edee, I. Fenniche, G. Granet, and B. Guizal, Prog. Electromagn. Res. 133, 17-35 (2013).

${ }^{7}$ K. Edee and B. Guizal, J. Opt. Soc. Am. A 30, 631-639 (2013).

${ }^{8}$ K. Edee and J.-P. Plumey, J. Opt. Soc. Am. A 32, 402-410 (2015).

${ }^{9}$ L. Verslegers, Z. Yu, Z. Ruan, P. B. Catrysse, and S. Fan, Phys. Rev. Lett. 108, 083902 (2012).

${ }^{10}$ M. Miyata, J. Hirohata, Y. Nagasaki, and J. Takahara, Opt. Express 22, 11399-11406 (2014). 